\title{
molecules
}

ISSN 1420-3049

www.mdpi.com/journal/molecules

Article

\section{The Feedback Regulation of PI3K-miR-19a, and MAPK-miR-23b/27b in Endothelial Cells under Shear Stress}

\author{
Jian He ${ }^{1,2}$, Yulin $\mathrm{Li}^{1{ }^{1} *}$, Xufang Yang ${ }^{3}$, Xu He ${ }^{1}$, Haiying Zhang ${ }^{1}$, Jin $\mathrm{He}^{4}$ and Lihong Zhang ${ }^{1}$ \\ 1 The Key Laboratory of Pathobiology, Ministry of Education, Norman Bethune College of Medicine, \\ Jilin University, Changchun 130021, China \\ 2 Departments of Bioengineering and Medicine and Institute of Engineering in Medicine, \\ University of California, San Diego, La Jolla, CA 92037, USA \\ 3 Department of Pathophysiology, Mudanjiang Medical College, Mudanjiang 157000, China \\ 4 The First Clinical Hospital, Jilin University, Changchun 130021, China \\ * Author to whom correspondence should be addressed; E-Mail: ylli@mail.jlu.edu.cn; \\ Tel.: +86-431-8561-9808.
}

Received: 16 November 2012; in revised form: 12 December 2012 / Accepted: 14 December 2012 /

Published: 20 December 2012

\begin{abstract}
Mechanical stimulation regulates endothelial cell (EC) functions through the modulation of signaling networks and gene expression. Our recent studies have identified that shear stress regulation of microRNAs (miRs)-19a, 23b and 27b, led to the modulation of EC proliferation. However, the underlying molecular mechanisms by which shear stress regulates these miRs have not been explored. Previous studies showed that shear stress activates multiple signaling pathways, including phosphatidylinositol 3 kinase (PI3K) and mitogen-activated protein kinase (MAPK). In this work we demonstrate that inhibition of the PI3K pathway attenuated the shear-induced miR-19a, and inhibition of the MAPK pathway attenuated miR-23b, 27b. The knockdown of miR-19a using antagomir-19a oligonucleotide (AM19a) decreased the shear-induced PI3K activation; whereas AM-23b, $27 \mathrm{~b}$ reduced the shear-induced MAPK activation. Furthermore, the overexpression of miR-19a overrode the suppressive effects of PI3K inhibitors on shear-induced PI3K activation; the overexpression of miR-23b, 27b had similar effects on ERK activations, but had little effect on P38 and JNK activation. Our findings suggest a positive feedback loop whereby PI3K and MAPK mediate the shear regulation of miR expression, which in turn modulates the shear-regulated PI3K/MAPK signaling events in ECs.
\end{abstract}


Keywords: endothelial cell; feedback loop; mechanical force (shear stress); microRNA; PI3 Kinase/MAP Kinase

\section{Selected Abbreviations and Acronyms:}

HUVECs human umbilical venous endothelial cells

MiRs microRNAs

PI3K phosphoinositide 3-kinase

MAPKs mitogen-activated protein kinases

ERK extracellular signal-regulated kinase

\section{Introduction}

Hemodynamic forces regulate the structure and function of the blood vessel wall, which was reported by Langille et al. [1]. Vascular endothelial cells (ECs), located at the interface between the circulating blood and the blood vessel, are exposed to shear stresses resulting from the tangential forces exerted by the flowing fluid on the vessel wall, leading to the modulation of signaling networks and expression of microRNAs [2-4]. ECs respond to changes of blood flow and distending pressure and convert mechanical stimuli into intracellular signals to affect cellular functions, e.g., proliferation, apoptosis, migration, permeability, and remodeling, as well as gene expression [3,5]. In the arterial tree, regional differences in shear stress forces produce distinct effects on the EC phenotype. Laminar shear, present in the straight portions of the tree, elicits a potential anti-inflammatory and atheroprotective response in ECs [6]. In previous studies, we focused on this atheroprotective shear stress force and found an upregulation of a distinct group of miRNAs that led to distinct functional consequences [7,8]. MicroRNAs (miRs) are short noncoding 18-24 nucleotide RNAs that negatively regulate the expression of target genes at the posttranscriptional level [9]. Among the mechano-sensetive miRs in ECs, atheroprotective shear stress induces miR-23b, 27b and 19a leads to EC growth arrest $[7,8]$. However, the mechanisms by which shear stresses regulate miR expression remain unexplored.

Previous studies showed that mechanical forces, exerted by fluid shearing, activate the phosphatidylinositol 3 (PI3) kinase and mitogen-activated protein (MAP) kinase pathways [10-13] and the shear-induced activations can be attenuated by specific chemical inhibitors [14-16]. Activation of PI3K and MAPK pathways has been implied to promote EC cell proliferation, migration and survival [10,17-19]. The role of miRs in PI3K and MAPK-modulated EC functions under shear remains undetermined.

In this work we found the inhibition of the PI3K pathway attenuated the shear-induced expression of miR19a, and inhibition of the MAPK pathway attenuated shear-induced miR-23b, 27b. Inhibition of miR-19a using antagomir-19a oligonucleotide (AM19a) diminished the shear-induced PI3K/AKT activation; similarly, inhibition of miR-23b, 27b using antagomir-23b oligonucleotide (AM23b) and antagomir-27b oligonucleotide (AM27b), respectively, reversed the shear-induced MAPK activation. Overexpression of miR-19a using pre-miR-19a significantly attenuated the blockade effects of PI3K 
inhibitor; likewise, overexpression of miR-23b, 27b significantly attenuated the blockade of MAPK inhibitor. Our findings indicate a feedback loop in which PI3K/AKT and MAPK mediate shearregulation of miRs expression, and miRs as well modulate PI3K/AKT and MAPK signaling in human ECs under shear conditions.

\section{Results}

\subsection{Inhibition of PI3K and MAPK Pathways Attenuated the Shear-Induction of miR-19a and miR-23b/27b, Respectively}

Using qPCR, we compared the expression of miRs in ECs after exposure to a laminar shear stress of 12 dyne $/ \mathrm{cm}^{2}$ for the indicated time periods with those cultured under static conditions for the same time periods. MiR-19a was significantly increased at $4 \mathrm{~h}(1.689 \pm 0.238$ fold in comparison to the time matched static control) after shearing. MiR-23b was significantly increased as early as $1 \mathrm{~h}$ ( $2.42 \pm 0.48$ fold $)$ and this lasted at least for $4 \mathrm{~h}$ (2.37 \pm 0.40 fold $)$; miR-27b was significantly increased (2.50 \pm 0.36 fold $)$ at $1 \mathrm{~h}$, and decreased to $1.37 \pm 0.27$ fold at $4 \mathrm{~h}$ (Figure 1). These results demonstrate that they were relatively early-responsive miRs to shear stress in ECs.

Figure 1. Shear stress regulation of endothelial cells. Laminar shear stress regulated miR expressions. QRT-PCR shows that laminar shear stress (12 dyne $\left./ \mathrm{cm}^{2}\right)$ significantly upregulated miR-19a at $4 \mathrm{~h}, \mathrm{miR}-23 \mathrm{~b}$ at $1 \mathrm{~h}$ and $4 \mathrm{~h}$, and miR-27b at $1 \mathrm{~h}$. $* p<0.05$ (compared with 1), \# $p<0.05$ between two time points. Data are mean $\pm \operatorname{SEM}(\mathrm{n}=6)$.

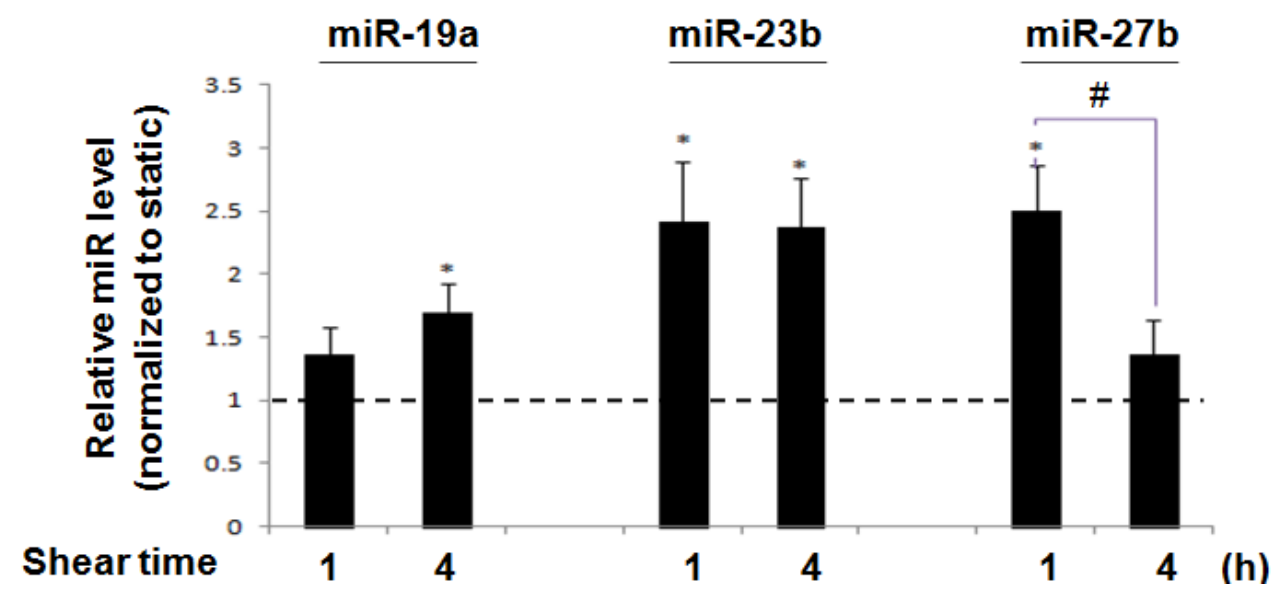

It has been shown that shear caused quick activations of PI3K/AKT and all three MAPKs [10-13]. We proceeded to investigate the roles of PI3K/AKT and MAPKs in shear-induction of miR expression. Under shear, treatments with LY294002 (PI3K inhibitor) and PD98059 (ERK inhibitor), SB203580 (p38 inhibitor) and SP600125 (JNK inhibitor) significantly attenuated the shear-induced phosphorylation of AKT, ERK1/2, P38, and JNK, respectively (Supplementary Figure S1). These results confirmed the inhibitory effects of these inhibitors on the flow-activation of each of their cognate kinases. Moreover, the inhibition of PI3K abolished shear-induced miR-19a expression, but had little effect on miR23b and 27b expression (Figure 2). The inhibition of ERK, JNK, or P38 attenuated the shear-induced miR-23b/27b expression, but not miR19a (Figure 2). These results identify the potential differential signaling pathways for shear-induced miR-19a and miR-23b/27b expressions. 
Figure 2. $\mathrm{AKT}$ and MAPK in shear-regulation of miRs. $10 \mu \mathrm{M}$ LY 294002 (PI3K inhibitor), PD 98059 (MAPK inhibitors), SB 203580 (pP38 inhibitor) and SP 600125 (pJNK inhibitor) were used to block the shear-induced phosphorylation of AKT, ERK1/2, p38 and JNK in ECs. 10 $\mu \mathrm{M}$ DMSO was employed as control. QRT-PCR shows the inhibition of PI3K pathway attenuated the shear-induced miR-19a, but there were no significantly changes of miR-23b and $27 \mathrm{~b}$; inhibition of MAPK pathway attenuated the shear-induced miR-23b and 27b, but not miR-19a. Data are presented as the mean \pm SEM $(\mathrm{n}=6) .{ }^{*} p<0.05$ (compared with the static control at the same time point).

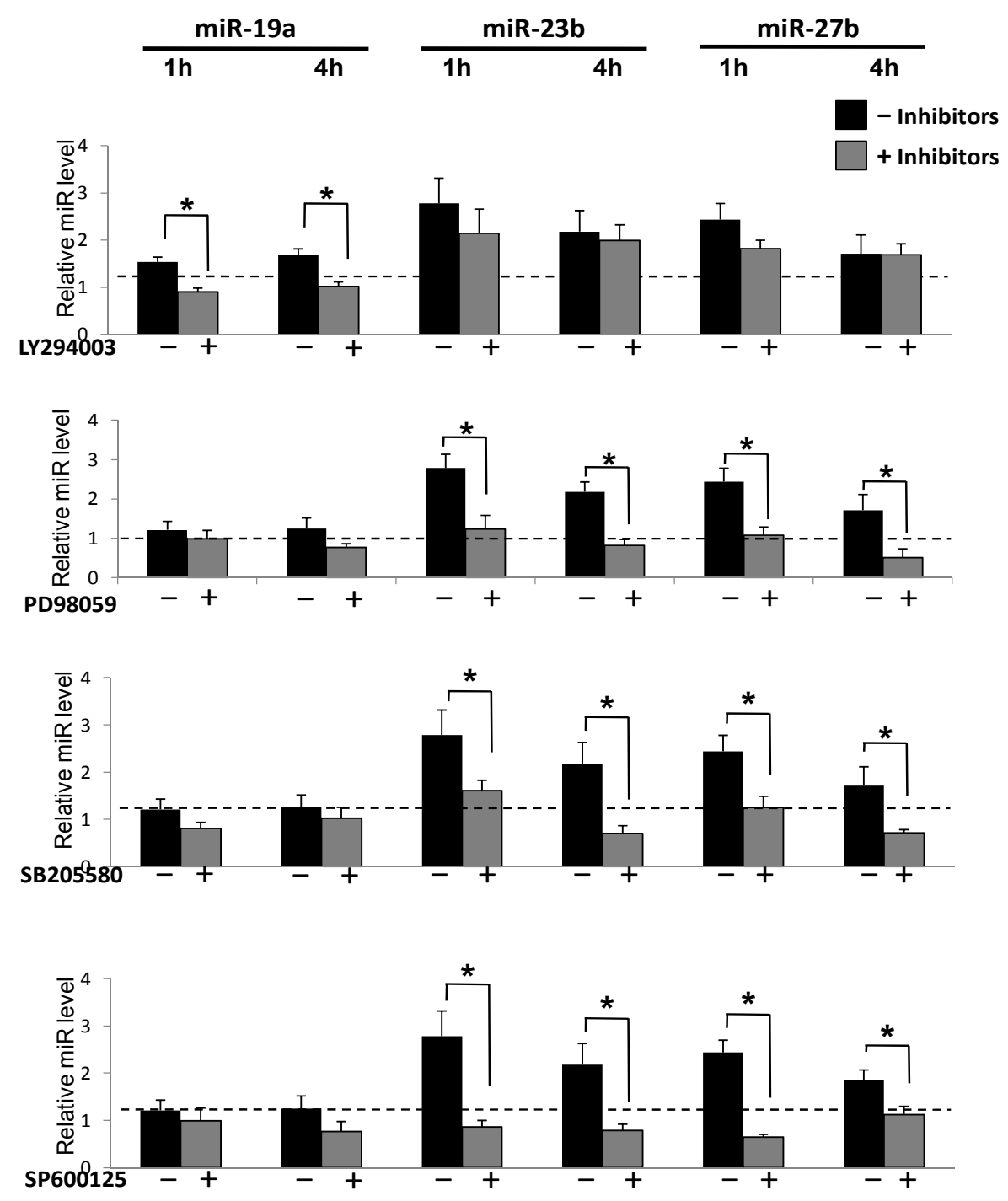

2.2. The Knockdown of miR-19a Decreased the Shear-Induced PI3K Activation; and Knockdown of miR-23b and miR-27b Reduced the Shear-Induced MAPK Activation

In order to investigate the interplay between miR expression and signaling pathways, we performed the loss-of-function analyses to study the effects of miR-19a, 23b and 27b knockdowns on shear-induced AKT and MAPK phosphorylation (Supplementary Figure S2). Antagomir against miR-19a, miR-23b and miR-27b (AM19a, 27b and 23b) were transfected into HUVECs to reduce the endogenous levels of these miRs. The target genes of miR-19a and 23b/27b are cyclin D1 (CCND1) and E2F1, respectively. The increased expression of the target genes indicated the AM transfection worked. 
Transfection of AM19a significantly decreased the shear-induced AKT activation (Figure 3A) in comparison the cells transfected with negative control AMCs. Transfection of AM23b and AM27b reduced the shear-induced MAPK activation (Figure 3B). These results suggest a feedback loop in which AKT mediated shear-induction of miR-19a, which may lead to a further increase of the shearinduced AKT activation. A similar feedback mechanism is also observed in MAPK-miR-23b/27b pathway.

Figure 3. Inhibition of miRs in shear-regulation of AKT and MAPK. (A) Antagomir against miR-19a decreased the shear-induced PI3K activation, but had no effect on MAPK activation; (B) Antagomir against miR-23b, 27b decreased the shear-induced MAPK activation, but had no effect on PI3K activation. Data are presented as the mean \pm SEM $(\mathrm{n}=3)$ (Data were normalized to the control in Neg Cont. group). ${ }^{*} p<0.05$ (Compared with the Static); in pAKT assay, ${ }^{\Delta} p<0.05$ (Compared with the shear groups in Neg Cont., AM23b and AM27b groups; in pERK, pP38, pJNK assay, ${ }^{\Delta} p<0.05$ (Compared with the shear groups in Neg Cont. and AM19a groups).

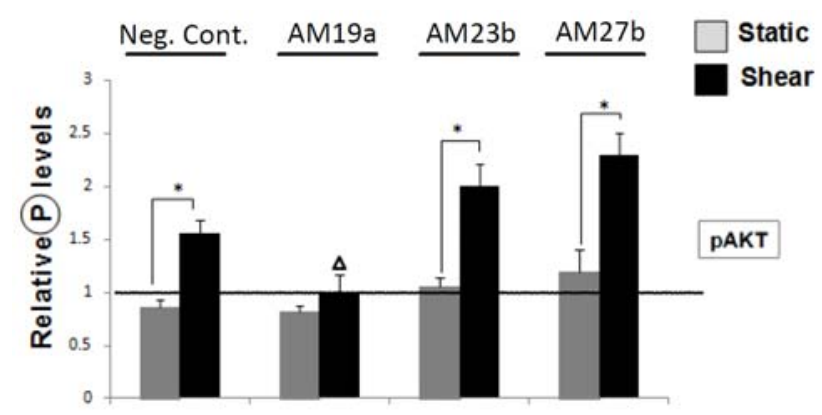

(A)

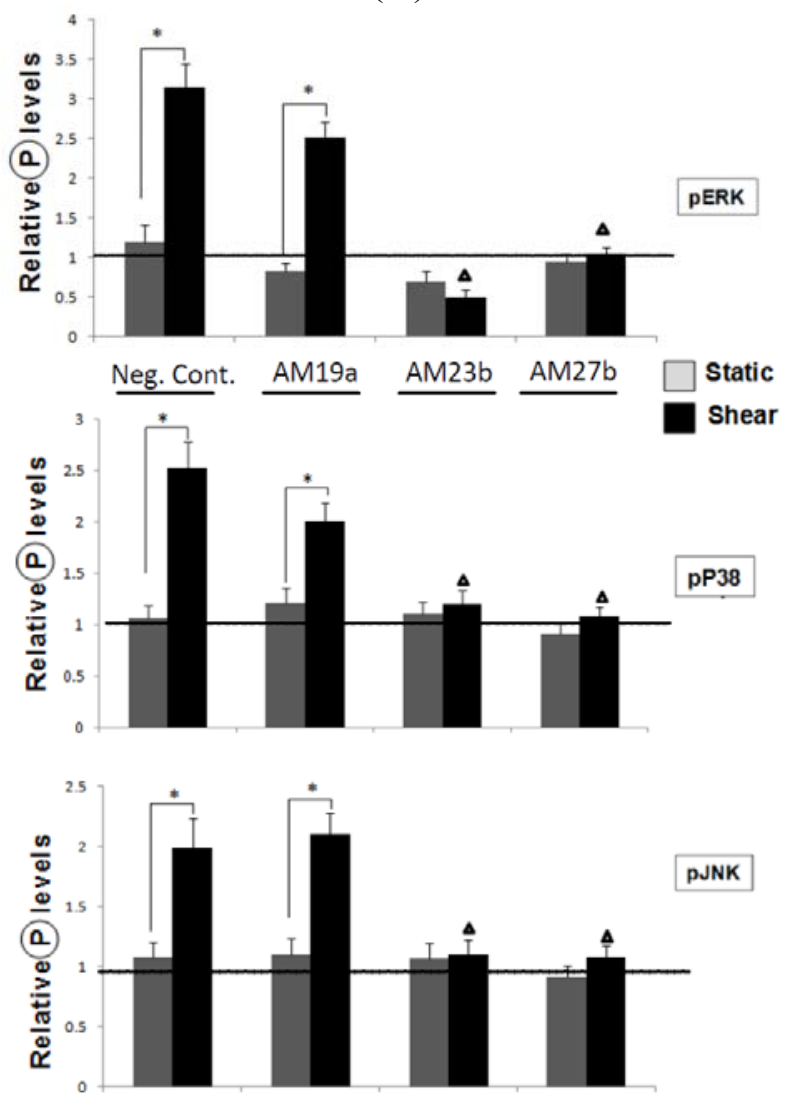

(B) 


\subsection{The Overexpression of miR-19a Attenuated the Suppressive Effects of PI3K Inhibitors on}

Shear-Induced PI3K/AKT Activation; the Overexpression of miR-23b, 27b Overrode the Inhibitory Effects of PD98059 on ERK Activations

To further establish the correlations between signaling pathways and miR expression under shear, we performed gain-of-functions analyses to investigate the effect of overexpression of miRs in shear-induced signaling. Precursor microRNAs (PreMiRs) were used to increase the expression levels of the corresponding miRs (Supplementary Figure S3). We found that the overexpression of miR-19a overrides the suppressive effects of AKT inhibitors on shear-induced AKT activation (Figure 4A); the overexpression of miR-23b, 27b had similar effects on shear-induced ERK activation (Figure 4B). However, PreMiR23b and 27b did not reverse the inhibitory effects of SB203580. PreMiR27b also overrode the inhibitory effects of SP600125 on shear-induced JNK phosphorylation. Notably, Pre-MiR23b and 27b slightly increased P38 and JNK phosphorylation under both static (ST) and sheared (SS) conditions (Figure 4B). These results demonstrated that miR-19a, miR-23b/27b play important roles in regulating EC signaling. Together with the data of loss-of-function of AM-19a, 23b and $27 \mathrm{~b}$ in laminar shear-induced PI3K and MAPK pathways, the results suggest a feedback loop of PI3K-miR-19a, and MAPK-miR23b/27b.

Figure 4. Overexpression of miRs in AKT and MAKP phosphorylation. (A) Transfection of miR-19a precursor enhanced AKT phosphorylation; (B) Transfection of miR-23b, 27b precursors overrode the suppressive effects of ERK inhibitors on shear-induced ERK, but not p38 and JNK, activation. Pre-MiR-27b overrode the SP600126 inhibition of JNK phosphorylation under shear. Data are presented as the mean $\pm \operatorname{SEM}(\mathrm{n}=3) .{ }^{*} p<0.05$.

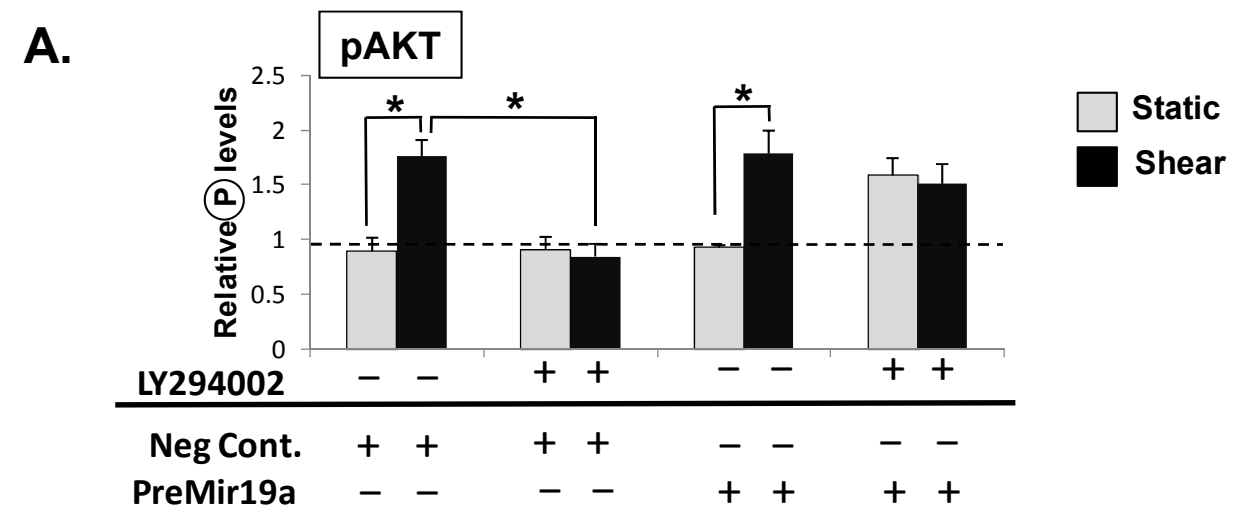


Figure 4. Cont.

B.
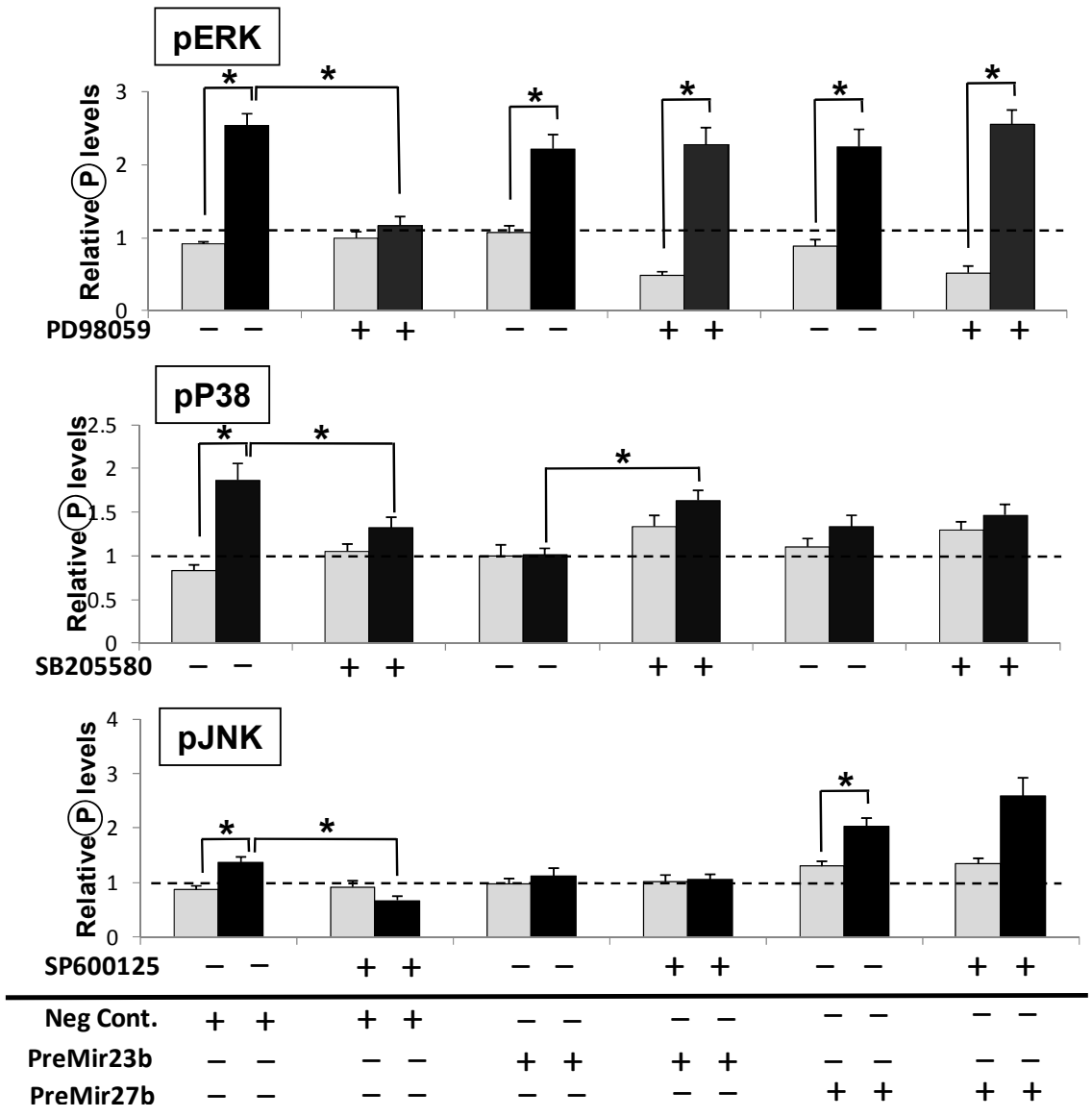

\section{Discussion}

MiR-19a consists of mir-17-92 microRNA clusters with miR-17, miR-18, miR-19b, miR-20, miR-25, miR-92, miR-93, miR-106a, and miR-106b [20], which are usually considered as oncogenic miRNA, which promotes cell proliferation and angiogenesis [21-23]. The bioinformatics analyses identify that miR-17-92 targets p21 that contributes to cell proliferation: targets PTEN/PI3K/AKT for anti-apoptotic effects; and targets CTGF and Tsp1 to promote angiogenesis [21]. Besides these target genes, it has been recently reported that TNF is one of the target genes in esophageal cancer development. The inhibition of miR-19a leads to esophageal cancer cell growth arrest and apoptosis [24]. However, it has been shown that miR-19a is induced by laminar shear stress to target cyclin D1 and this led to an attenuation of the G1/S transition in ECs [8], indicating the role of miR-19a is beyond oncogenesis and cell cycle angiogenesis.

The PI3K/AKT pathway plays an important role in many cellular functions. Activation of $\mathrm{PI} 3 \mathrm{~K} / \mathrm{AKT}$ pathway signaling leads to the mTOR-mediated tumor angiogenesis $[25,26]$. In particular, PI3K involves in multiple EC functions, e.g., promoting EC proliferation, migration and survival [26]. Both PI3K and miR-19a has been shown to be involved in EC mechanotransduction [10,27]. The role of the PI3K pathway in regulating miR19a is not examined. Our current study showed that the inhibition of PI3K pathway attenuated the shear-induced miR19a, the knockdown of miR19a using AM19a decreased the shear-induced PI3K activation and the overexpression of miR-19a enhanced the AKT phosphorylation in ECs treated with LY 294002, and attenuated its suppressive effects on shear- 
induced PI3K activation. These results suggest a possible feedback loop whereby PI3K mediates shear regulation of miR expression.

MiR23b and 27b are members of the MiR-23-27-24 cluster, which is highly expressed in many vascularized tissues, and play critical roles in cardiovascular development, angiogenesis, and EC homeostasis [28]. Recently it is reported that miR-27b controls endothelial tip cell fate, branching, and venous specification by determining Spry2 (Sprouty homologue 2) and D114 (Delta-like ligand 4) as its essential targets.

The inhibition of Spry leads to the activation of MAPK according to the literature. This is another piece of strong evidence to demonstrate that miR-27b plays important roles in regulating EC signaling [29]. MiR-23b and 27b were shown to be induced by shear stress [2,7,30], and MiR23b played an important role in shear-induced G2/M EC growth arrest [7]. Shear-induced G2/M arrest and the corresponding changes in G2/M regulatory protein expression through Smad 1/5 have been reported [31]. MiR-23b targeting Smads (Smad3, Smad4 and Smad5) serve as a molecular switch in regulating transforming growth factor- $\beta 1$ (TGF- $\beta 1$ ) in regulating liver stem cell differentiation [32], a model in which miR-23b clusters promote the growth of fetal hepatocytes by down-regulating Smads and consequently TGF- $\beta$ signaling [30]. MAPK-mediated phosphorylation of Smads 2 and 3 may confer resistance to TGF mediated growth inhibition in cancer cells harboring a hyperactive Ras/MAPK pathway [33]. One report has shown that TGF induces expression of miR23 cluster (miRs-23a, 27a, and 24) in Huh7 cells through a Smads dependent mechanism [34]. Akiko Hata's [35] group showed that Smad proteins control Drosha-mediated microRNA-21 maturation and Smad proteins bind a conserved RNA sequence to promote microRNA maturation by Drosha [36]. Here, we have shown that the Smad upstream signaling molecules, MAPKs, are involved in the shear-induced miR23b/27b expressions, and miR-23b/27b also played a feedback role in controlling shear-induced MAPK activation. The role of Smads in mediating shear-regulated MAPK-miR loop remains to be determined. In addition, it is interesting that the overexpression of miR23b/27b with PreMiRs, overrode the PD98095 inhibition of shear-induced ERK phosphorylation, which suggests that miR23b/27b may be targeting some negative regulator for ERKs, however the overexpression of $\mathrm{miR} 23 \mathrm{~b} / 27 \mathrm{~b}$ did not resume the shear-inducibility for P38 and JNK after treatment with inhibitors, suggesting that miR23b/27b may play a more important role in ERK signaling. It is also interesting to note that AM23b caused an elevation of ERK phosphorylation under static condition; and PreMiR23b/27b reduced ERK phosphorylation under static condition with PD98095 treatment. These results strongly suggest that miR23b/27b targeting negative regulators for MAPKs. More studies will be needed to elucidate the miR23b/27b targets for MAPK signalings.

In summary, we demonstrated feedback loops that MAPK and PI3K were involved in the regulation of miRs expression in ECs by shear stress. These results revealed a mechanism by which mechanical forces modulate the interplay of miR and mechanotransduction network in ECs.

\section{Material and Methods}

\subsection{Cell Culture and Reagents}

HUVECs were isolated from human umbilical cord veins as previously described [37]. The cells were cultured in Medium 199 (Invitrogen, Carlsbad, CA, USA) supplemented with 10\% fetal bovine 
serum (Omega), 10\% endothelial cell growth medium (Cell Applications, San Diego, CA, USA), 1\% L-glutamine, $1 \%$ sodium pyruvate, and 1\% penicillin/streptomycin. All cell cultures were kept in a humidified $5 \% \mathrm{CO}_{2} / 95 \%$ air incubator at $37{ }^{\circ} \mathrm{C}$. Cells within passages 5 to 6 were used in all experiments. The chemical inhibitors LY 294002, PD 98059, SB 203580 and SP 600125 all at the work concentration of $10 \mu \mathrm{M}$ (EMD Millipore Chemical, San Diego, CA, USA), were used to block the activities of PI3K, ERK, p38 and JNK respectively. Before flow experiments, the cells were subjected $12 \mathrm{~h}$ low serum condition $(0.5 \%$ FBS $)$ followed by the pretreatments with the blocking reagents for $1 \mathrm{~h}$ to inhibit the specific kinase functions [15].

\subsection{Shear Stress Experiments}

A parallel-plate flow chamber device was used as an in vitro system to study the responses of cultured ECs to shear stresses at the cellular and molecular levels as described before [38]. In brief, HUVECs cultured on $38 \times 76-\mathrm{mm}$ slides and assembled into a chamber in that a flow channel was created by using a gasket with a rectangular cutout with uniform channel height along the flow path. HUVECs in the rectangular channel are exposed to laminar shear stress (SS) at $12 \mathrm{dyne} / \mathrm{cm}^{2}$ generated by a pressure difference between the inlet and the outlet of the chamber (Figure 5). The flow system was maintained at $37{ }^{\circ} \mathrm{C}$ in a hood and equilibrated with $5 \% \mathrm{CO}_{2} / 95 \%$ air.

Figure 5. Side view of the laminar flow chamber. The endothelial cell (EC) monolayers cultured to confluence on the bottom surface of the rectangular channel are exposed to the flow generated by a pressure difference between the inlet and the outlet of the chamber. The flow system is maintained at $37^{\circ} \mathrm{C}$ in a hood and equilibrated with $5 \% \mathrm{CO}_{2} / 95 \%$ air. The insert in channel shows an enlarged view of two ECs and the flow profile of the applied shear stress.

\section{Side view of laminar flow chamber}

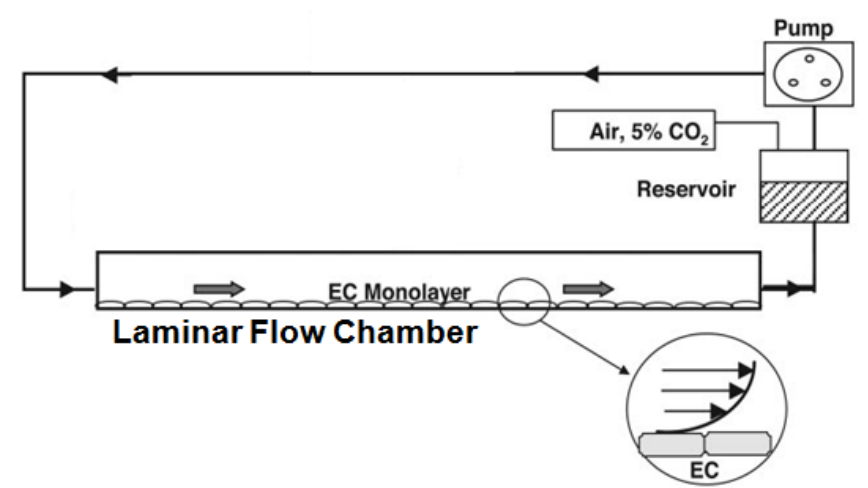

\subsection{MicroRNA RT-qPCR}

Quantitative assessment of specific miR levels was performed using the TaqMan miR assay kits (Applied Biosystems, Carlsbad, CA, USA) according to the manufacturer's protocol. MiR cycle threshold $(\mathrm{Ct})$ values were normalized to internal control (RNU48) and converted into copy numbers, by $\Delta \Delta C T$ method [39], and shear groups were then normalized to the time match static controls. 


\subsection{Western Blot Analysis}

Western Blot analysis was performed using standard protocols. Antibodies against pAKT, total AKT, pERK1/2, pP38, P38 were obtained from Cell Signaling, and anti-GAPDH, ERK2, pJNK, JNK2 were obtained from Santa Cruz (Santa Cruz, CA, USA). These were used in a 1:1,000 dilution, and an HRP-conjugated secondary antibody, followed by enhanced chemiluminescence detection.

\subsection{Pre-mir and Antagomir Transfection}

Premirs were transfected into ECs to increase the expression levels of the corresponding miRs; antagomirs against miR-19a and $23 \mathrm{~b} / 27 \mathrm{~b}$, as well as negative control molecule (Anti-miR ${ }^{\mathrm{TM}}$ miRNA Inhibitor Negative Control \#1), were purchased from Ambion (Carlsbad, CA, USA). The oligonucleotides were individually transfected with siPort NeoFx reagent (Ambion, Carlsbad, CA, USA) at the final concentration of $50 \mathrm{nM}, 12-\mathrm{h}$ post-transfection. Using qPCR to detect the expression of the target genes of miR-19a and 23b/27b, CCND1 and E2F1resepctively after transfected $12 \mathrm{~h}$. The increased expression of the target genes indicated the transfection of AM worked, and the decreased expression of the target genes meant the Premirs transfection worked.

\section{Conclusions}

Our findings suggest a positive feedback loop in which PI3K and MAPK mediate shear regulation of miR expression, which in turn modulates the shear-regulated PI3K/MAPK signaling events in ECs.

\section{Supplementary Materials}

Supplementary materials can be accessed at: http://www.mdpi.com/1420-3049/18/1/1/s1.

\section{Acknowledgments}

This work was supported by National Key Program of Basic Research Development (973 Program) Project No. 81172499; National Natural Science Foundation of China No. 30470662; Key Progect of Jilin Provincial Science \& Technology Department, No. 20076023 from Yulin Li and a scholarship from the China Scholarship Council for Jian He.

\section{References}

1. Langille, B.L. Arterial remodeling: Relation to hemodynamics. Can. J. Physiol. Pharmacol. 1996, 74, 834-841.

2. Boon, R.A.; Hergenreider, E.; Dimmeler, S. Atheroprotective mechanisms of shear stress-regulated microRNAs. Thromb. Haemost. 2012, 108, 616-620.

3. Li, Y.S.; Haga, J.H.; Chien, S. Molecular basis of the effects of shear stress on vascular endothelial cells. J. Biomech. 2005, 38, 1949-1971.

4. Chien, S. Mechanotransduction and endothelial cell homeostasis: The wisdom of the cell. Am. J. Physiol. Heart Circ. Physiol. 2007, 292, H1209-H1224. 
5. Lin, K.; Hsu, P.P.; Chen, B.P.; Yuan, S.; Usami, S.; Shyy, J.Y.; Li, Y.S.; Chien S. Molecular mechanism of endothelial growth arrest by laminar shear stress. Proc. Natl. Acad. Sci. USA 2000, 97, 9385-9389.

6. Shyu, K. Cellular and molecular effects of mechanical stretch on vascular cells and cardiac myocytes. Clin. Sci. 2009, 116, 377-389.

7. Wang, K.C.; Garmire, L.X.; Young, A.; Nguyen, P.; Trinh, A.; Subramaniam, S.; Wang, N.P.; Shyy, J.J.; Li, Y.S.; Chien, S. Role of microRNA-23b in flow-regulation of Rb phosphorylation and endothelial cell growth. Proc. Natl. Acad. Sci. USA 2010, 107, 3234-3239.

8. Qin, X.; Wang, X.; Wang, Y.; Tang, Z.; Cui, Q.; Xi, J.; Li, J.Y.; Chien, S.; Wang, N. MicroRNA19a mediates the suppressive effect of laminar flow on cyclin D1 expression in human umbilical vein endothelial cells. Proc. Natl. Acad. Sci. USA 2010, 107, 3240-3244.

9. Sun, W.; Li, Y.S.; Huang, H.D.; Shyy, J.Y.; Chien, S. MicroRNA: A Master Regulator of Cellular Processes for Bioengineering Systems. Annu. Rev. Biomed. Eng. 2010, 12, 1-27.

10. Dimmeler, S.; Assmus, B.; Hermann, C.; Haendeler, J.; Zeiher, A.M. Fluid shear stress stimulates phosphorylation of Akt in human endothelial cells: Involvement in suppression of apoptosis. Circ. Res. 1998, 833, 34-41.

11. Tseng, H.; Peterson, T.E.; Berk, B.C. Fluid shear stress stimulates mitogen-activated protein kinase in endothelial cells. Circ. Res. 1995, 77, 869-878.

12. Li, Y.S.; Shyy, J.Y.; Li, S.; Lee, J.; Su, B.; Karin, M.; Chien, S. The Ras-JNK pathway is involved in shear-induced gene expression. Mol. Cell. Biol. 1996, 16, 5947-5954.

13. Jalali, S.; Li, Y.S.; Sotoudeh, M.; Yuan, S.; Li, S.; Chien, S.; Shyy, J.Y. Shear stress activates p60 src-Ras-MAPK signaling pathways in vascular endothelial cells. Arterioscler. Thromb. Vasc. Biol. 1998, 18, 227-234.

14. Alessi, D.R.; Cuenda, A.; Cohen, P. PD098059 is a specific inhibitor of the activation of Mitogenactivated protein kinase in vitro and in vivo. J. Biol. Chem. 1995, 270, 27489-27494.

15. Wu, C.C.; Li, Y.S.; Haga, J.H.; Wang, N.; Lian, I.Y.; Su, F.C. Roles of MAP Kinases in the Regulation of Bone Matrix Gene Expressions in Human Osteoblasts by Oscillatory Fluid Flow. J. Cell. Biochem. 2006, 98, 632-641.

16. Bennett, B.L.; Sasaki, D.T.; Murray, B.W.; O’Leary, E.C.; Sakata, S.T.; Xu, W. SP600125, an anthrapyrazolone inhibitor of Jun N-terminal kinase. Proc. Natl. Acad. Sci. USA 2001, 98, 13681-13686.

17. Dimmeler, S.; Zeiher, A.M. Akt takes center stage in angiogenesis signaling. Circ. Res. 2000, 86, 4-5.

18. Dimmeler, S.; Dernbach, E.; Zeiher, A.M. Phosphorylation of the endothelial nitric oxide synthase at ser-1177 is required for VEGF induced endothelial cell migration. FEBS Lett. 2000, $477,258-262$.

19. Rikitake, Y.; Kawashima, S.; Yamashita, T.; Ueyama, T.; Ishido, S.; Hotta, H.; Hirata, K.; Yokoyama, M. Lysophosphatidylcholine inhibits endothelial cell migration and proliferation via inhibition of the extracellular signal-regulated kinase pathway. Arterioscler. Thromb. Vasc. Biol. 2000, 20, 1006-1012.

20. Tanzer, A.; Stadler, P.F. Molecular evolution of a microRNA cluster. J. Mol. Biol. 2004, 339, $327-335$. 
21. Olive, V.; Jiang, I.; He, L. mir-17-92, a cluster of miRNAs in the midst of the cancer network. Int. J. Biochem. Cell Biol. 2010, 42, 1348-1354.

22. Kuehbacher, A.; Urbich, C.; Dimmeler, S. Targeting microRNA expression to regulate angiogenesis. Trends Pharmacol. Sci. 2008, 29, 12-15.

23. He, J.; He, X.; Zhang, H.; Yang, X.; He, J.; Zhang, Li.; Li, Y. Effect of Mesima Reishi UE-1 on invasion of human ovarian cancer cells in vitro. Afr. J. Biotechnol. 2011, 10, 9888-9897.

24. Biyashev, D.; Veliceasa, D.; Topczewski, J.; Topczewska, J.M.; Mizgirev, I.; Vinokour, E.; Reddi, A.L.; Licht, J.D.; Revskoy, S.Y.; Volpert, O.V. miR-27b controls venous specification and tip cell fate. Blood 2012, 119, 2679-2687.

25. Advani, S.H. Targeting mTOR pathway: A new concept in cancer therapy. Indian J. Med. Paediatr. Oncol. 2010, 4, 132-136.

26. Yuan, R.; Kay, A.; Berg, W.J.; Lebwohl, D. Targeting tumorigenesis: Development and use of mTOR inhibitors in cancer therapy. J. Hematol. Oncol. 2009, 2, doi:10.1186/1756-8722-2-45.

27. Go, Y.M.; Park, H.; Maland, M.C.; Darley-Usmar, V.M.; Stoyanov, B.; Wetzker, R.; Jo, H. Phosphatidylinositol 3-kinase gamma mediates shear stress-dependent activation of JNK in endothelial cells. Am. J. Physiol. 1998, 275, H1898-H1904.

28. Bang, C.; Fiedler, J.; Thum, T. Cardiovascular importance of the microRNA-23/27/24 family. Microcirculation 2012, 19, 208-214.

29. Roy, S.; Khanna, S.; Hussain, S.R.; Biswas, S.; Azad, A.; Rink, C.; Gnyawali, S.; Shilo, S.; Nuovo, G.J.; Sen, C.K. MicroRNA expression in response to murine myocardial infarction: miR-21 regulates fibroblast metalloprotease-2 via phosphatase and tensin homologue. Cardiovasc. Res. 2009, 82, 21-29.

30. Urbich, C.; Kaluza, D.; Frömel, T.; Knau, A.; Bennewitz, K.; Boon, R.A.; Bonauer, A.; Doebele, C.; Boeckel, J.N.; Hergenreider, E.; et al. MicroRNA-27a/b controls endothelial cell repulsion and angiogenesis by targeting semaphorin 6A. Blood 2012, 119, 1607-1616.

31. Chang, S.F.; Chang, C.A.; Lee, D.Y.; Lee, P.L.; Yeh, Y.M.; Yeh, C.R.; Cheng, C.K.; Chien, S.; Chiu, J.J. Tumor cell cycle arrest induced by shear stress: Roles of integrins and Smad. Proc. Natl. Acad. Sci. USA 2008, 105, 3927-3932.

32. Rogler, C.E.; Levoci, L.; Ader, T.; Massimi, A.; Tchaikovskaya, T.; Norel, R.; Rogler, L.E. MicroRNA-23b cluster microRNAs regulate transforming growth factor-beta/bone morphogenetic protein signalling and liver stem cell differentiation by targeting Smads. Hepatology 2009, 50, 575-554.

33. Massagué, J. Integration of Smad and MAPK pathways: A link and a linker revisited. Genes Dev. 2003, 17, 2993-2997.

34. Huang, S.; He, X.; Ding, J.; Liang, L.; Zhao, Y.; Zhang, Z.; Yao, X.; Pan, Z.; Zhang, P.; Li, J.; et al. Upregulation of miR-23a, 27a, 24 decreases transforming growth factor-beta-induced tumorsuppressive activities in human hepatocellular carcinoma cells. Int. J. Cancer 2008, 123, 972-978.

35. Davis, B.N.; Hilyard, A.C.; Lagna, G.; Hata, A. SMAD proteins control DROSHA-mediated microRNA maturation. Nature 2008, 454, 56-61.

36. Davis, B.N.; Hilyard, A.C.; Nguyen, P.H.; Lagna, G.; Hata, A. Smad proteins bind a conserved RNA sequence to promote microRNA maturation by Drosha. Mol. Cell 2010, 39, 373-384. 
37. Gimbrone, M.A., Jr.; Cotran, R.S.; Folkman, S.B.J. Human vascular endothelial cells in culture. Growth and DNA synthesis. J. Cell Biol. 1974, 60, 673-684.

38. Frangos, J.A.; Eskin, S.G.; McIntire, L.V.; Ives, C.L. Flow effects on prostacyclin production by cultured human endothelial cells. Science 1985, 227, 1477-1479.

39. Chen, C.; Ridzon, D.A.; Broomer, A.J.; Zhou, Z.; Lee, D.H.; Nguyen, J.T.; Barbisin, M.; Xu, N.L.; Mahuvakar, V.R.; Andersen, M.R.; et al. Real-time quantification of microRNAs by stem-loop RT-PCR. Nucleic Acids Res. 2005, 33, e179.

Sample Availability: Not available.

(C) 2013 by the authors; licensee MDPI, Basel, Switzerland. This article is an open access article distributed under the terms and conditions of the Creative Commons Attribution license (http://creativecommons.org/licenses/by/3.0/). 